\title{
Isolation And Characterization Of 38 SNP Markers For The Black Rockfish, Sebastes Schlegelii By Next- Generation Sequencing
}

\author{
Xianggang Gao ( $\sim$ xiangganggao@163.com )

\section{Xiangbo Bao} \\ Liaoning Ocean and Fisheries Science Research Institute \\ Wei Sun \\ Liaoning Ocean and Fisheries Science Research Institute

\section{Yunfeng Li} \\ Liaoning Ocean and Fisheries Science Research Institute

\section{Zongying Liu} \\ Liaoning Ocean and Fisheries Science Research Institute \\ Weidong Liu \\ Liaoning Ocean and Fisheries Science Reseach Institute
}

Liaoning Ocean and Fisheries Science Research Institute https://orcid.org/0000-0002-6757-5701

\section{Research Article}

Keywords: Sebastes schlegelii, SNP, RAD, Genetic diversity

Posted Date: June 7th, 2021

DOI: https://doi.org/10.21203/rs.3.rs-247269/v1

License: (c) (1) This work is licensed under a Creative Commons Attribution 4.0 International License. Read Full License

Version of Record: A version of this preprint was published at Conservation Genetics Resources on July 27th, 2021. See the published version at https://doi.org/10.1007/s12686-021-01226-3. 


\section{Abstract}

The black rockfish (Sebastes schlegelii) is an economically important species. However, wild S. schlegelii resources have declined sharply in recent years as a result of human disturbance and habitat destruction. Thus, it is crucial to protect the current resources of $S$. schlegelii. In this study, 38 novel single nucleotide polymorphism (SNP) markers were developed based on restriction-site associated DNA sequencing. The results showed that the observed heterozygosity and expected heterozygosity ranged from 0.1400 to 0.6400 and 0.1487 to 0.4978 , respectively. The minor allele frequency raged from 0.1429 to 0.4694 . Polymorphic information content ranged from 0.174 to 0.365. Four SNPs were found to be deviated significantly from the HWE $(P<0.05)$. These SNP markers will serve as a useful tool for genetic studies and population evaluation aimed at the conservation of $S$. schlegelii.

\section{Main Text}

The black rockfish (Sebastes schlegelii) is an important commercial fish distributed in Northwest Pacific enjoying high popularity in China, Korea and Japan (Yoshida et al. 2005). In recent decades, commercial exploitation and environmental changes have caused a decline in its population. Thus, demand for hypervariable molecular markers to provide a population-genetic perspective on conservation and management efforts of the species (FAO, 1993) becomes urgent.

With the rapid development of next-generation sequencing technologies (NGS) (Davey et al. 2011), single nucleotide polymorphisms (SNPs) have been largely developed and widely used for genetic studies in aquaculture species such as Megalobrama terminalis (Yang et al. 2020), Coilia ectenes (Yu et al. 2019) and Ochetobius elongatus (Yang et al. 2018). For $S$. schlegelii resource conservation, we implemented restriction-site associated DNA (RAD) sequencing to facilitate the genetic evaluation.

In this study, a total of $50 \mathrm{~S}$. schlegelii wild individuals were collected from northern Yellow Sea in China. Muscle tissues were sampled and stored in 95\% molecular grade ethanol. Total genomic DNA was extracted from tissue samples using the TIANamp Marine Animals DNA Kit (Tiangen, Beijing, China) following the manufacturer's instructions. 20 samples were used to constructed the RAD libraries. Then, the libraries were sequenced on the Illumina HiSeq 4000 platform using 150 base pair (bp) paired-end reads. We trimmed the adapter sequences and low-quality reads (Phred score < 20) with Cutadapt (Martin 2011). Finally, 3,014,591 putative SNPs with the highest scores were generated, from which we randomly selected 90 candidate SNPs to test their applicability. The polymorphism of these candidate SNPs was further characterized in the remaining 30 samples mentioned above. Primer sequences for SNP loci were designed by Primer 5.0 software. The PCR reactions were conducted in $25 \mu \mathrm{L}$ volume containing $50 \mathrm{ng}$ of genomic DNA, $1 \times$ PCR buffer, $1.5 \mathrm{mM} \mathrm{MgCl}_{2}, 0.2 \mathrm{mM}$ dNTPs, $200 \mathrm{nM}$ of each primer, and $1 \mathrm{U}$ of Taq polymerase (Takara, Dalian, China). Amplicons were checked by $1.0 \%$ agarose gel electrophoresis and sequenced on ABI 3730 DNA Analyzer (Applied Biosystems).

The observed heterozygosity $(\mathrm{Ho})$, expected heterozygosity $(\mathrm{He})$, minor allele frequency (MAF), and $\mathrm{P}$ value representing the deviations from the Hardy-Weinberg equilibrium were estimated using POPGENE 32 (Yeh et al. 2007). The polymorphism information content (PIC) was calculated using Cervus 3.0 (Kalinowski et al. 2007). Among the test SNP markers in S. schlegelii, 38 polymorphic SNP markers were characterized in Table 1. The Ho and He were ranged from 0.1400 to 0.6400 and 0.1487 to 0.4978 , respectively. The MAF raged from 0.1429 to 0.4694 . The PIC varied from 0.174 to 0.365 , with an average of 0.286 . Four SNPs were found to be deviated significantly from the HWE $(P<0.05)$. These results will be useful for understanding the genetic diversity of $S$. schlegelii to assist in the management of this germplasm resource. 
Table 1 Summary information for the 38 SNP markers developed for the S. schlegelii 


\begin{tabular}{|c|c|c|c|c|c|c|c|c|}
\hline $\begin{array}{l}\text { Primer } \\
\text { ID }\end{array}$ & Primer sequences & $\begin{array}{l}\text { SNP } \\
\text { type }\end{array}$ & $\begin{array}{l}\text { Size } \\
\text { (bp) }\end{array}$ & Ho & $\mathrm{He}$ & MAF & PIC & $P_{\mathrm{HWE}}$ \\
\hline \multirow[t]{2}{*}{ HYF1 } & F: TATCTGAGGTTTGGCTCCCAC & $\mathrm{G} / \mathrm{A}$ & 335 & 0.3400 & 0.2851 & 0.2093 & 0.198 & 0.1617 \\
\hline & R:CTCACTTGCTTAAGGTCATTCAGG & & & & & & & \\
\hline \multirow[t]{2}{*}{ HYF6 } & F: AAGACATTCAGGTCCAGCTCTG & $\mathrm{C} / \mathrm{T}$ & 401 & 0.6400 & 0.4396 & 0.3600 & 0.341 & 0.0011 \\
\hline & R: AGCAAACCCTTGACTTTCTGGC & & & & & & & \\
\hline \multirow[t]{2}{*}{ HYF8 } & F: GAATACACCCACCTGTCACTC & $A / G$ & 447 & 0.2800 & 0.2982 & 0.2917 & 0.258 & 0.6586 \\
\hline & R: AGCTTTCTTCAACTCAAAGTGG & & & & & & & \\
\hline \multirow[t]{2}{*}{ HYF9 } & F: ATGAATTGACAAGAATAGCCTG & $\mathrm{C} / \mathrm{T}$ & 414 & 0.3000 & 0.3982 & 0.3333 & 0.332 & 0.7655 \\
\hline & R: CATATGTTTCAGCGACTCCA & & & & & & & \\
\hline \multirow[t]{2}{*}{ HYF11 } & F: ACTGCTGGCAACTAGTTCATGG & $\mathrm{T} / \mathrm{C}$ & 278 & 0.2800 & 0.2715 & 0.2857 & 0.226 & 0.8202 \\
\hline & R: TGCTGTTGAGGACACACGG & & & & & & & \\
\hline \multirow{2}{*}{ HYF13 } & F: AAGCAGTCATGAGGCTTGAG & G/A & 426 & 0.2600 & 0.2576 & 0.2826 & 0.236 & 0.9453 \\
\hline & R: TAGTCAATGCCATGGTTGGC & & & & & & & \\
\hline \multirow[t]{2}{*}{ HYF14 } & F: TTCTGGTTCCAGTCAGCGTC & $A / G$ & 388 & 0.4200 & 0.3788 & 0.2927 & 0.265 & 0.4339 \\
\hline & R: CAGTACCAGAAGATTCAGCACAC & & & & & & & \\
\hline \multirow[t]{2}{*}{ HYF18 } & F: ATCATTTCCGAGCAGTGACC & $\mathrm{C} / \mathrm{A}$ & 304 & 0.3200 & 0.2982 & 0.3200 & 0.252 & 0.2812 \\
\hline & R: CATCAGTGAATGAAAGTGGTGC & & & & & & & \\
\hline \multirow[t]{2}{*}{ HYF23 } & F: AACACTTTTGGACACAAGCTCC & $\mathrm{C} / \mathrm{T}$ & 378 & 0.4400 & 0.3887 & 0.4082 & 0.308 & 0.3427 \\
\hline & R: AATGGTGATGCAAGTTCGCTG & & & & & & & \\
\hline \multirow[t]{2}{*}{ HYF25 } & F: AGCTAAGGTCAAGGTGATGC & G/A & 333 & 0.1400 & 0.4160 & 0.1429 & 0.335 & 0.0000 \\
\hline & R: AGAACTATACAGGAGAGGTGCC & & & & & & & \\
\hline \multirow[t]{2}{*}{ HYF26 } & F: ATAGTGGTCAGCTGCATCAAG & $\mathrm{G} / \mathrm{A}$ & 287 & 0.4400 & 0.4655 & 0.4082 & 0.354 & 0.6956 \\
\hline & R: GACTGATCATACCGACGAGTC & & & & & & & \\
\hline \multirow[t]{2}{*}{ HYF27 } & F: AGCTGTCTACACTAGGTCCAG & $T / C$ & 417 & 0.1600 & 0.1487 & 0.2162 & 0.174 & 0.5670 \\
\hline & R: TAGGCCTCTGTTATCTTGGGTC & & & & & & & \\
\hline \multirow[t]{2}{*}{ HYF29 } & F: CAGATTGATGTGAGTGGTCCTG & $\mathrm{C} / \mathrm{G}$ & 389 & 0.3400 & 0.3982 & 0.3400 & 0.317 & 0.2939 \\
\hline & R: ATGACATCCACCTCGTCTTGG & & & & & & & \\
\hline \multirow[t]{2}{*}{ HYF30 } & F: GATTGTAGGGCTGCAGTTTGTC & $A / T$ & 344 & 0.1800 & 0.1655 & 0.1633 & 0.153 & 0.5116 \\
\hline & R: ATAGCTCTGCCACAGTCAATGG & & & & & & & \\
\hline \multirow[t]{2}{*}{ HYF33 } & F: CTCACTCCTATTACAGTGCAGAG & $T / C$ & 308 & 0.4000 & 0.3685 & 0.4000 & 0.298 & 0.5381 \\
\hline & R: GTCTCGTAACTGTAGAAGGACC & & & & & & & \\
\hline HYF34 & F: TTTGTCTCTCCAGTCGCTGG & $\mathrm{C} / \mathrm{T}$ & 411 & 0.4600 & 0.3578 & 0.4000 & 0.269 & 0.3956 \\
\hline
\end{tabular}


R: TCTCTGTGGTACCTGTAGCG

\begin{tabular}{|c|c|c|c|c|c|c|c|c|}
\hline \multirow[t]{2}{*}{ HYF35 } & F: TGAGATGGTTGTTCAGTTCAGAC & \multirow[t]{2}{*}{$\mathrm{G} / \mathrm{A}$} & \multirow[t]{2}{*}{325} & \multirow[t]{2}{*}{0.1400} & \multirow[t]{2}{*}{0.1978} & \multirow[t]{2}{*}{0.2800} & \multirow[t]{2}{*}{0.177} & \multirow[t]{2}{*}{0.0310} \\
\hline & R: TAGAACATGACAGAGCATGCAG & & & & & & & \\
\hline \multirow[t]{2}{*}{ HYF37 } & F: TCTGTTAGTCTTCCACTGCTG & \multirow[t]{2}{*}{$\mathrm{G} / \mathrm{A}$} & \multirow[t]{2}{*}{305} & \multirow[t]{2}{*}{0.3000} & \multirow[t]{2}{*}{0.2851} & \multirow[t]{2}{*}{0.3478} & \multirow[t]{2}{*}{0.256} & \multirow[t]{2}{*}{0.7034} \\
\hline & R: ATGCCAGTATCTTCAGTCTACC & & & & & & & \\
\hline \multirow[t]{2}{*}{ HYF39 } & F: CAAATGTCAAAGTGTCCTCGG & \multirow[t]{2}{*}{$A / G$} & \multirow[t]{2}{*}{270} & \multirow[t]{2}{*}{0.4400} & \multirow[t]{2}{*}{0.3685} & \multirow[t]{2}{*}{0.4082} & \multirow[t]{2}{*}{0.295} & \multirow[t]{2}{*}{0.1624} \\
\hline & R: ATTTCACACCTCACTATGACGG & & & & & & & \\
\hline \multirow[t]{2}{*}{ HYF42 } & F: TCTCAGCTTTACAGTGATACCC & \multirow[t]{2}{*}{$\mathrm{C} / \mathrm{T}$} & \multirow[t]{2}{*}{436} & \multirow[t]{2}{*}{0.4200} & \multirow[t]{2}{*}{0.4596} & \multirow[t]{2}{*}{0.3913} & \multirow[t]{2}{*}{0.347} & \multirow[t]{2}{*}{0.5375} \\
\hline & R: GAAATGCTAGAGTGCGTCTG & & & & & & & \\
\hline \multirow[t]{2}{*}{ HYF47 } & F: TACAGACTGTTCTCATTCAGGG & \multirow[t]{2}{*}{$\mathrm{C} / \mathrm{G}$} & \multirow[t]{2}{*}{311} & \multirow[t]{2}{*}{0.3800} & \multirow[t]{2}{*}{0.3109} & \multirow[t]{2}{*}{0.3800} & \multirow[t]{2}{*}{0.252} & \multirow[t]{2}{*}{0.1078} \\
\hline & R: GAGTTCAGAGTGATTGACAGC & & & & & & & \\
\hline
\end{tabular}

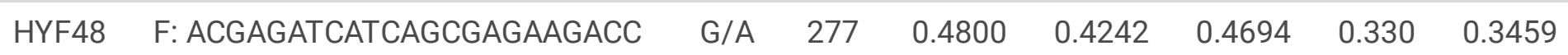
R: TTCCACCGTTTGACCACTACG

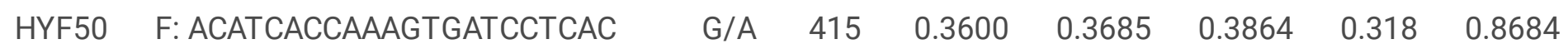
R: ACATTCAAGCTCACAGAGGC

$\begin{array}{lllllllll}\text { HYF65 F: ACAAACAGCGGTCTCCTGAG } & \text { A/T } & 321 & 0.3000 & 0.3578 & 0.3000 & 0.298 & 0.2447\end{array}$ R: ccacctgtgttcagttacagtc

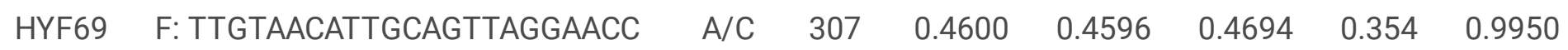
R: gcataactttgtagaagggacc

$\begin{array}{llllllll}\text { HYF70 F: TAACATTGCAGTTAgGAACCTC } & \text { T/C } & 389 & 0.3200 & 0.4242 & 0.3404 & 0.331 & 0.0781\end{array}$ R: ttgagtcattggtgtaattctacc

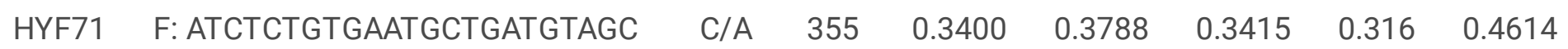
R: atgcaatgcactgtactcctg

$\begin{array}{lllllllll}\text { HYF74 F: } & \text { G/A } & 383 & 0.3600 & 0.3887 & 0.3600 & 0.311 & 0.5958\end{array}$

TGAGGGCATAACCCATGCTGTAGC

R:tgacaaagtggcagtatgtgacgag

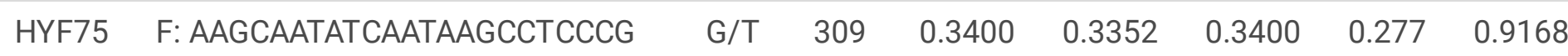
R: gacaaagtggcagtatgtgacg

$\begin{array}{llllllll}\text { HYF76 F: TACTTCCCTCTGCTGCAGTC } & \text { T/G } & 301 & 0.3600 & 0.4978 & 0.3750 & 0.368 & 0.0480\end{array}$

R: tagcaaatccaccatgtcctacc

$\begin{array}{lllllllll}\text { HYF77 F: TCTCAGATTTGGAGGTAGCCAC } & \text { C/G } & 441 & 0.2600 & 0.2576 & 0.2889 & 0.228 & 0.9453\end{array}$

R: tcatttggcactttgtgtccg

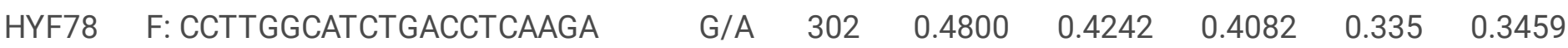




\begin{tabular}{|c|c|c|c|c|c|c|c|c|}
\hline & R: AAcaatactgctggttctgctagg & & & & & & & \\
\hline HYF79 & F: AACCAATGAGGAAACTCCAACAG & $A / G$ & 286 & 0.3000 & 0.2851 & 0.3000 & 0.242 & 0.7034 \\
\hline & R: cttgttccatttaggtgagccag & & & & & & & \\
\hline HYF80 & F: TATCACCGATACCCAATCCAG & $\mathrm{C} / \mathrm{T}$ & 271 & 0.4200 & 0.3982 & 0.4000 & 0.311 & 0.6939 \\
\hline & R: tggagttaaagagtgtccagtc & & & & & & & \\
\hline HYF81 & $\begin{array}{l}\text { F: } \\
\text { TGAAGACAGAGCTAGTGTAGATGGG }\end{array}$ & $\mathrm{C} / \mathrm{T}$ & 239 & 0.2400 & 0.2715 & 0.2857 & 0.261 & 0.3986 \\
\hline & R: tgataagtgcatccgactctgc & & & & & & & \\
\hline HYF85 & $\begin{array}{l}\text { F: } \\
\text { AGATCTGACAGAGATTCCAATCGG }\end{array}$ & $\mathrm{T} / \mathrm{A}$ & 311 & 0.3400 & 0.3352 & 0.3400 & 0.277 & 0.9168 \\
\hline & R: gcaatagtagttctcaagctatggg & & & & & & & \\
\hline HYF86 & $\begin{array}{l}\text { F: } \\
\text { TGCCATCATTGGTTCACATTAGGC }\end{array}$ & $\mathrm{C} / \mathrm{T}$ & 224 & 0.4000 & 0.4848 & 0.4000 & 0.365 & 0.2109 \\
\hline & R: agtttggctgtttggtcatgcg & & & & & & & \\
\hline HYF90 & $\begin{array}{l}\text { F: } \\
\text { ACTGTTCGTGAAGCTCAAACTCAG }\end{array}$ & $\mathrm{T} / \mathrm{A}$ & 428 & 0.3600 & 0.3685 & 0.3750 & 0.305 & 0.8684 \\
\hline & R: agaaattccgctaagggcagc & & & & & & & \\
\hline
\end{tabular}

Ho observed heterozygosity, He expected heterozygosity, MAF minor allele frequency, PIC polymorphism information content, $P_{\mathrm{HWE}}$ the $P$ value of the deviations from the Hardy-Weinberg equilibrium

\section{Declarations}

Author contributions G.X.G. conceived and designed the study, contributed to the drafted the manuscript. B.X.B. contributed to the analysis and writing. S.W. participated in the data collection, purification, and management of the samples for sequencing and genotyping. L.W.D. contributed to the design of the study and writing. L.Y.F. and L.Z.Y. contributed to the collection of the samples. All authors have reviewed and approved the manuscript.

Funding This study was funded by the Agricultural Major Project of Liaoning Province (2020JH1/10200002), Foundation of President of Liaoning Academy of Agricultural Sciences (2021QN2425), and the Special Funding for Modern Agricultural Industrial Technology System (CARS-47-Z04).

Availability of data The sequence data used for SNP discovery belongs to Liaoning Ocean and Fisheries Science Institute, and it can be available upon reasonable request.

Conflict of interest The authors declare that they have no conflict of interest.

Ethical approval Investigations were conducted according to the guiding principles for the use and care of laboratory animals and in compliance with the Academic Ethics Committee of Liaoning Ocean and Fisheries Science Institute. Our study had been submitted to and approved by the Academic Ethics Committee of Liaoning Ocean and Fisheries Science Institute. The research permits also included the necessary ethical approval in terms of sample collection. All sample collection was undertaken in accordance with relevant Academic Ethics Committee of Liaoning Ocean and Fisheries Science Institute guidelines and regulations. 
Consent to participate Not applicable

Consent for publish Not applicable

\section{References}

1. Davey JW, Hohenlohe PA, Etter PD, Boone JQ, Catchen JM, Blaxter ML (2011) Genome-wide genetic marker discovery and genotyping using next-generation sequencing. Nat Rev Genet 12(7):499-510.

https://doi.org/10.1038/nrg3012

2. FAO (1993). Report of the expert consultation on utilization and conservation of aquatic genetic resources. FAO Fish Rep. 491: 1-58

3. Kalinowski ST, Taper ML, Marshall TC (2007) Revising how the computer program CERVUS accommodates genotyping error increases success in paternity assignment. Mol Ecol 16:1099-1106

4. Martin M (2011) Cutadapt removes adapter sequences from high throughput sequencing reads. EMBnet $J$ 17(1):10-12.https://doi.org/10.14806/ej.17.1.200

5. Yang JP, Li YF, Zhu SL, Chen WT, Li J, Xue HM, Li XH (2018) Development and characterization of 26 SNP markers in Ochetobius elongatus based on restriction site-associated DNA sequencing (RAD-seq). Conserv Genet Resour. https://doi.org/10.1007/ s12686-018-1075-3

6. Yang JP , Li XH , Li YF, Zhu SL, Chen WT, Li J (2020) Isolation and characterization of 30 SNP markers in Guangdong bream (Megalobrama terminalis) by next-generation sequencing. Conserv Genet Resour. https://doi.org/10.1007/s12686-020-01131-1

7. Yeh FC, Yang R, Boyle TJ, Ye Z, Xiyan JM (2000) POPGENE 32, Microsoft Windows-based freeware for population genetic analysis. Molecular Biology and Biotechnology Centre, University of Alberta, Edmonton

8. Yoshida K, Nakagawa M and Wada S (2005). Multiplex PCR system applied for analyzing microsatellite loci of Schlegel's black rockfish, Sebastes schlegeli. Mol Ecol Res. 5 (2): 416-418. https://doi.org/10.1111/j.14718286.2005.00945.x

9. Yu AQ, Shi YH, Yan YL (2019) Development and characterization of 50 snp markers in Coilia ectenes. Conserv Genet Resour. https:// doi.org/10.1007/s12686-019-01086-y 